\title{
Prostate biopsy free system for laparoscopic radical prostatectomy in a pituitary dwarfism: a case report
}

\author{
Jiatong Zhou, Baoling Zhang, Shuai Xia, Tao Li, Ranlu Liu \\ Department of Urology, The Second Hospital of Tianjin Medical University, Tianjin, China \\ Correspondence to: Ranlu Liu. Department of Urology, The Second Hospital of Tianjin Medical University, No. 23, Pingjiang Road, Hexi District, \\ Tianjin 300211, China. Email: 16622080858@163.com.
}

\begin{abstract}
Prostate biopsy is the gold standard for the diagnosis of prostate cancer. However, not all patients are suitable for prostate biopsy. For example, some patients have anal stenosis, some patients are too old to withstand the pain caused by puncture, patients who are unwilling to undergo prostate biopsy. We found that there was currently no literature report on a specific solution to this problem. This is the first report of a laparoscopic radical prostatectomy (LRP) in a pituitary dwarfism who didn't have a prostate biopsy before LRP due to anal stenosis. And this report added a new method to diagnose prostate cancer. We present a case of a 61-year-old pituitary dwarfism who had a prostate specific antigen (PSA) of $32.13 \mathrm{ng} / \mathrm{mL}$ by physical examination and didn't perform prostate biopsy due to anal stenosis. Preoperative prostate MRI suggests a low-signal mass on the left side of the prostate and ${ }^{68} \mathrm{Ga}$ PSMA-11 PET/CT demonstrated that Abnormally high PSMA and CHO uptake on the left side of the prostate. Therefore, combined with the patient's PSA, MRI and ${ }^{68} \mathrm{Ga}$ PSMA-11 PET/CT, our clinical diagnosis was prostate cancer. Surgery was difficult due to narrow pelvic space, but achievable through LRP. Histological analysis revealed multifocal prostate cancer, with negative surgical margins and no extraprostatic extension. Postoperative patient had no serious complications and was discharged. Based on this case, For the first time, we proposed to make full use of the results of clinical tests and imaging examinations for the diagnosis and treatment of diseases without prostate biopsy.
\end{abstract}

Keywords: Prostate cancer; prostatectomy; pituitary dwarfism; case report

Submitted Jan 28, 2020. Accepted for publication Sep 18, 2020.

doi: $10.21037 /$ tau-20-489

View this article at: http://dx.doi.org/10.21037/tau-20-489

\section{Introduction}

Although the current diagnosis of prostate cancer mainly depends on prostate biopsy, there were still some special reports demonstrated that prostate biopsy might be not a significant diagnosis of prostate cancer. For example, a case report showed that a patient underwent twice prostate biopsies, and the results were all negative, and the metastatic lesion of the tumor appeared on the imaging. The biopsy of metastatic lesion finally confirmed prostate cancer (1), and another case reported that patient similarly underwent twice prostate biopsies and the results were negative, and they also showed distant metastasis on imaging. After long- term ADT treatment, the patient's bone pain symptoms eased and PSA decreased significantly (2) which showed this patient got prostate cancer.

Therefore, there are still some special cases for the diagnosis of prostate cancer. This is the first report of laparoscopic radical prostatectomy (LRP) in pituitary dwarfism who with no preoperative prostate biopsy due to anal stenosis. We highlighted a case of significantly clinical diagnosis of prostate cancer treated with LRP, and outline surgical and anesthetic considerations before proceeding in patients with pituitary dwarfism. We present the following article in accordance with the CARE reporting checklist (available at http://dx.doi.org/10.21037/tau-20-489). 


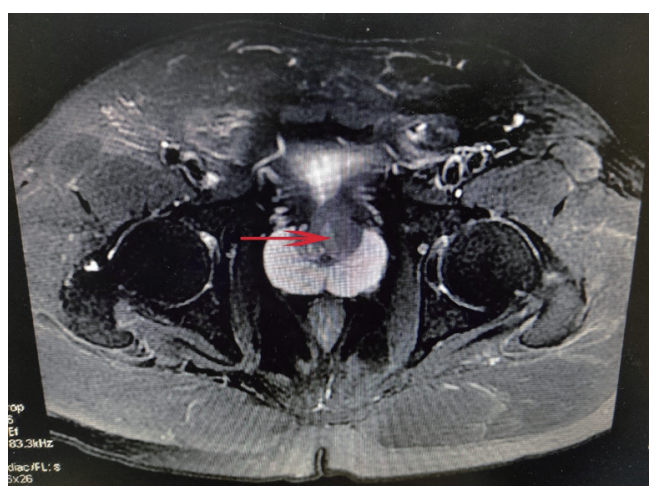

Figure 1 Prostate MRI in the pituitary dwarfism a narrow pelvis and short rectal diameter. Red arrow indicates a prostate mass.

\section{Case presentation}

A 61-year-old pituitary dwarfism presented with moderate lower urinary tract symptoms. 4 years ago, prostate specific antigen (PSA) level in this patient was $15 \mathrm{ng} / \mathrm{mL}$ by physical examination, and the patient rechecked PSA level one month ago and the PSA was $31.19 \mathrm{ng} / \mathrm{mL}$. During hospitalization, his PSA level was $32.13 \mathrm{ng} / \mathrm{mL}$. There was no history of genetic disease and prostate cancer in his family. His digital rectal exam (DRE) was failed to perform. This brought us a big problem that the patient's anus is too narrow to perform prostate biopsy. Because of the unusual anatomy and prostate biopsy failed, prostate MRI (Figure 1) and ${ }^{68}$ Ga PSMA-11 PET/CT (Figure 2) were undertaken and the results were negative for metastases. And we only found abnormal signal in central zone of prostate and no other abnormality, multidisciplinary review occurred. Our radiation oncologists and physicists demonstrated that it was consistent with the performance of prostate cancer according to the PSA of the patient and imaging findings. Therefore, in the absence of a clear histopathology, after multidisciplinary discussions, we combined PSA, MRI, PET-CT to diagnose patients with prostate cancer. From the image, the mass was confined to the prostate tissue and did not break through the capsula. At present, radical prostatectomy (RP) is one of the main treatment methods for localized prostate cancer. Our radiation oncologists and physicists suggested that RP to be the most appropriate treatment. Finally, we successfully removed the prostate and bilateral seminal vesicles by LRP. Histological analysis revealed unifocal adenocarcinoma (Gleason 3+5), with negative surgical margins, no lymphovascular invasion, and no extraprostatic extension. No obvious discomfort and stable vital signs after LRP. Post-operatively, patients received adjuvant hemotherapy, followed by PSA test. No major complication was reported after 3 months. The full timeline was presented shown in Figure 3.

\section{Discussion}

Patients with pituitary dwarfism, who have a human growth hormone (hGH) deficiency, appear child-like even as adults, with normally proportioned limbs and trunk, adenohypophysary hypofunction occurs if there is parenchymal loss of $75 \%$ or more. The causes of these patients may be congenital or acquired (3). The diameter of pelvis is narrow in patients with dwarfism. For women with dwarfism, the pelvic diameters of persons with pituitary dwarfism are usually only adequate for delivery of a preterm infant (4). Therefore, for patients with dwarfism, the narrow pelvic space greatly increases the difficulty of surgery, especially laparoscopic surgery.

Related health problems for consideration for anesthetics include cardiac and respiratory difficulties, cervical vertebral instability in achondroplastic dwarf $(5,6)$. Hence, we thought that pituitary dwarfism has increased mortality compared to the normal population in surgery. Franco et al. demonstrated that pituitary dwarfism there may be various anomalies of the dental apparatus, from the morphological profile and in terms of development (7). Patients with dwarfism due to various reasons need to be more cautious during anesthesia, and for patients with pituitary dwarfism, most people have a proportionately smaller airway without anatomic abnormalities $(3,8)$.

In this case, patient with dwarfism had certain risks in the process of anesthesia, so we should assess the risk of anesthesia well before surgery. Because of the congenital pelvic stenosis, there was the possibility of damaging the pelvic blood vessels during the operation, which brought us great difficulty in laparoscopic surgery. On the other hand, due to the patients with congenital anal stenosis who could not perform prostate biopsy successfully, which brought us great difficulty in clinical diagnosis. Therefore, we proposed a concept of prostate biopsy free. Through imaging diagnosis such as prostate MRI, ${ }^{68} \mathrm{Ga}$ PSMA-11 PET/CT, we could carry out clinical diagnosis of prostate cancer. After consultation with the patient and their families, we decided to carry out LRP for this patient, and the operation was successful. Finally, histological analysis confirmed that our preoperative diagnosis was accurate. But 


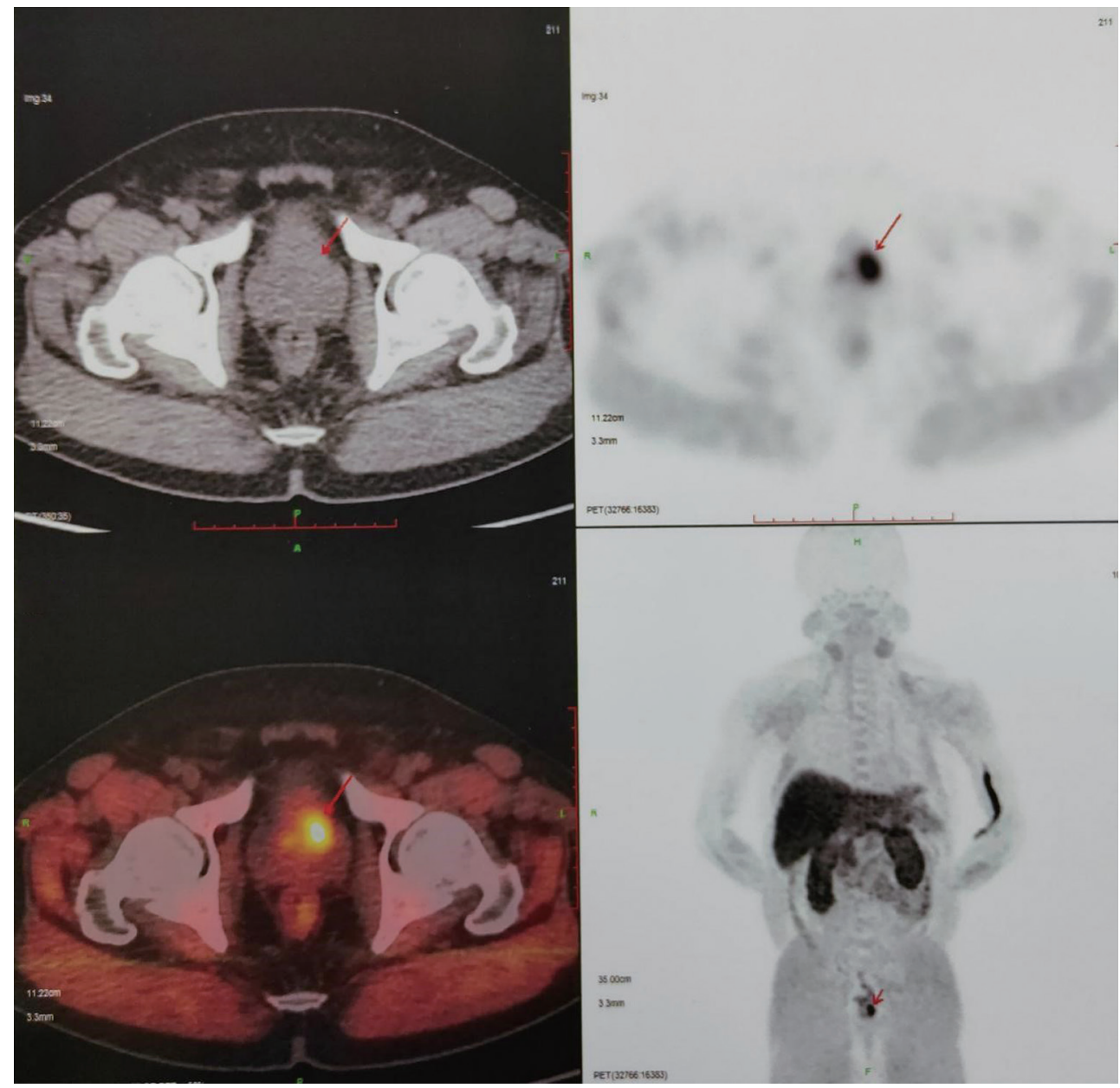

Figure $2{ }^{68} \mathrm{Ga}$ PSMA-11 PET/CT in the pituitary dwarfism. Red arrow indicates a prostate mass.

because of the lack of pathological diagnosis before surgery, this method was not suitable for all patients. We also found some rare case reports showing patients with bone pain and very high PSA who experienced twice prostate biopsy were negative results, and finally through the diagnosis method for bone metastases or lymph node biopsy to confirm the diagnosis $(1,9,10)$. Compared with their cases, we found the difference that our patients did not have symptoms of bone pain, PSA was not high, and there was no tumor metastasis or lymphadenopathy on the MRI, and prostate biopsy could not be completed. Iwamura et al. proposed a case that a 64-year-old man with PSA level of 2,036 ng/mL underwent twice prostate biopsies, and the results were negative. And the Imaging showed bone metastases, but no matestatic lesion biopsy was performed. After ADT, the patient's bone pain was alleviated, and PSA level was reduced. Therefore, they clinically diagnosed patients with prostate cancer (2).
Therefore, for these patients with negative biopsy and high suspicion of prostate cancer with distant metastasis, we could also diagnose prostate cancer through clinical diagnosis and used tentative ADT treatment to confirm the disease. For our case, we did not find distant metastases, and tumor was inside the prostate by imaging examination and no enlarged lymph nodes were found, so there was a risk of misdiagnosis. We needed to combine the indicators of this patient to improve the accuracy of diagnosis. For localized prostate cancer, radical prostatectomy (RP) is the preferred treatment. At present, the surgical methods of RP are LRP and robotic-assisted LRP. For this patient, we used LRP to excision of prostate. Although we knew that for patients with pelvic stenosis, RALP was superior to LRP in the choice of surgery, but due to the limitations of our surgical equipment, we still decided to complete the surgery through LRP. There was no lymph node metastasis was found in 


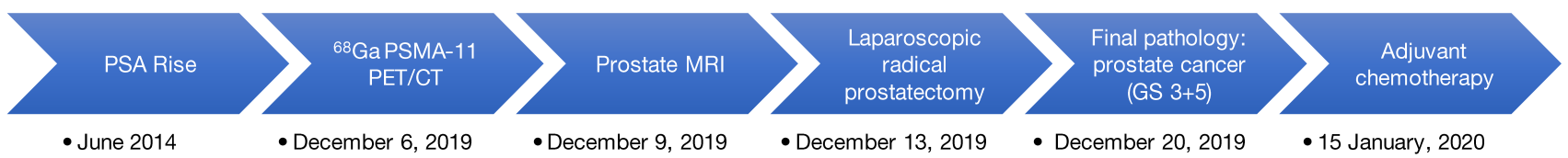

Figure 3 Timeline of examination and treatment.

the pathology of the patient, and the image did not show systemic metastasis at the same time. The postoperative pathology showed high-risk prostate cancer, so it was necessary to receive endocrine therapy and monitor PSA levels.

For this case, our diagnosis was successful, and the tumor was removed in time to improve patient survival. The strength of this case is that for patients who cannot undergo prostate biopsy and there is no metastasis and lymphadenopathy on imaging, we combined PSA, MRI, and PET-CT to make a diagnosis to avoid missing the presence of prostate malignant tumors. The biggest limitation in this case was the risk of misdiagnosis. In this case, no clear clinical information was obtained to indicate malignant tumors such as metastatic lesion or suspicious lymph nodes. we did not have specific data to enhance the accuracy of our diagnosis of prostate cancer in this case. Therefore, we hope to provide this case to solve the clinical problems. At the same time, we also hope to be able to design a scoring system to perform data analysis of laboratory data and subjective performance of different imaging studies, so that some patients can avoid unnecessary biopsy. And some patients who cannot be biopsied can also be clinically diagnosed.

All procedures performed in studies involving human participants were in accordance with the ethical standards of the institutional and/or national research committee(s) and with the Helsinki Declaration (as revised in 2013). Written informed consent was obtained from the patient.

\section{Conclusions}

Through this case, we can better use laboratory data and imaging tests to diagnose prostate cancer. Although it cannot replace prostate biopsy, for patients who are unable to perform prostate biopsy or are unwilling to perform prostate biopsy, we hope to apply the concept of prostate biopsy free in clinic.

\section{Acknowledgments}

Funding: The study was supported by Tianjin Science and
Technology Committee (19ZXDBSY00050).

\section{Footnote}

Reporting Checklist: The authors have completed the CARE reporting checklist. Available at http://dx.doi.org/10.21037/ tau-20-489

Conflicts of Interest: All authors have completed the ICMJE uniform disclosure form (available at http://dx.doi. org/10.21037/tau-20-489). The authors have no conflicts of interest to declare.

Ethical Statement: The authors are accountable for all aspects of the work in ensuring that questions related to the accuracy or integrity of any part of the work are appropriately investigated and resolved. All procedures performed in studies involving human participants were in accordance with the ethical standards of the institutional and/or national research committee(s) and with the Helsinki Declaration (as revised in 2013). Written informed consent was obtained from the patient.

Open Access Statement: This is an Open Access article distributed in accordance with the Creative Commons Attribution-NonCommercial-NoDerivs 4.0 International License (CC BY-NC-ND 4.0), which permits the noncommercial replication and distribution of the article with the strict proviso that no changes or edits are made and the original work is properly cited (including links to both the formal publication through the relevant DOI and the license). See: https://creativecommons.org/licenses/by-nc-nd/4.0/.

\section{References}

1. Ueda Y, Higuchii $Y$, Hashimoto T, et al. Prostate cancer diagnosed through the biopsy of the bone metastatic lesion; a case report. Hinyokika Kiyo 2007;53:327-30.

2. Mitsui $Y$, Sadahira T, Maruyama $Y$, et al. Burned-out Prostate Cancer? Primary Metastatic Cancer Not Detected on Repeat Biopsy. Acta Med Okayama 2018;72:605-9. 
3. Ratner EF, Hamilton CL. Anesthesia for cesarean section in a pituitary dwarf. Anesthesiology 1998;89:253-4.

4. Tyson JE, Barnes AC, McKusick VA, et al. Obstetric and gynecologic considerations of dwarfism. Am J Obstet Gynecol 1970;108:688-704.

5. Matsui Y, Kawabata H, Ozono K, et al. Skeletal development of achondroplasia: analysis of genotyped patients. Pediatr Int 2001;43:361-3.

6. Wynn J, King TM, Gambello MJ, et al. Mortality in achondroplasia study: a 42-year follow-up. Am J Med Genet A 2007;143A:2502-11.

7. Ferrante F, Blasi S, Crippa R, et al. Dental Abnormalities

Cite this article as: Zhou J, Zhang B, Xia S, Li T, Liu R. Prostate biopsy free system for laparoscopic radical prostatectomy in a pituitary dwarfism: a case report. Transl Androl Urol 2020;9(6):2831-2835. doi: 10.21037/tau-20-489 in Pituitary Dwarfism: A Case Report and Review of the Literature. Case Rep Dent 2017;2017:5849173.

8. Berkowitz ID, Raja SN, Bender KS, et al. Dwarfs: pathophysiology and anesthetic implications. Anesthesiology 1990;73:739-59.

9. Shimizu K, Nakano S, Okada Y, et al. Hinyokika Kiyo 2019;65:75-80.

10. Iwamura H, Hatakeyama S, Tanaka $Y$, et al. A case of metastatic cancer with markedly elevated PSA level that was not detected by repeat prostate biopsy. BMC Res Notes 2014;7:64. 